\title{
Cranial metastatic alveolar rhabdomyosarcoma mimicking hematological malignancy in an adolescent boy
}

\author{
Turkan Patiroglu • Bilgen Isik • Ekrem Unal • \\ Ozlem Canoz • Kemal Deniz • Kemal Kosemehmetoglu • \\ Musa Karakukcu • Mehmet Akif Ozdemir
}

Received: 10 May 2014 / Accepted: 16 May 2014 / Published online: 11 June 2014

(C) Springer-Verlag Berlin Heidelberg 2014

\begin{abstract}
Background Widespread alveolar rhabdomyosarcoma (ARMS) with bone marrow involvement and with an unknown primary tumor, especially presenting with acute tumor lysis syndrome can be easily misdiagnosed as a hematological malignancy. Furthermore, brain metastasis of ARMS is rare seen in children.

Case report Herein, we report a 14-year-old boy presenting with acute tumor lysis syndrome due to bone marrow invasion
\end{abstract}

T. Patiroglu • B. Isik $\cdot$ E. Unal $(\bowtie) \cdot$ M. Karakukcu $\cdot$ M. A. Ozdemir Division of Pediatric Hematology and Oncology, Department of Pediatrics, Faculty of Medicine, Erciyes University, 38039 Talas, Kayseri, Turkey

e-mail: drekremunal@yahoo.com.tr

E. Unal

e-mail: ekremunal@erciyes.edu.tr

T. Patiroglu

e-mail: turkanp@erciyes.edu.tr

B. Isik

e-mail: dr.bilgenayan@gmail.com

M. Karakukcu

e-mail: mkkukcu@yahoo.com

M. A. Ozdemir

e-mail: makifo@erciyes.edu.tr

O. Canoz $\cdot$ K. Deniz

Department of Pathology, Faculty of Medicine, Erciyes University,

Talas, Kayseri, Turkey

O. Canoz

e-mail: ocanoz@erciyes.edu.tr

K. Deniz

e-mail: drkdeniz@yahoo.com

K. Kosemehmetoglu

Department of Pathology, Faculty of Medicine, Hacettepe University, Ankara, Turkey

e-mail: dokemal@hotmail.com of ARMS, who was diagnosed after abdominal paraaortic lymph node biopsy. Despite radiological and nuclear medicine imaging, the primary tumor site could not be found. He was treated with vincristine, topotecan, and cyclophosphamide for 42 weeks. Six months after the completion of treatment, he suffered from severe headache, blurred vision, right hemiplegia, and severe bone pain. Cranial magnetic resonance imaging showed multiple hemorrhagic infarctions. Brain biopsy showed brain metastasis with PAX3-FKHR fusion transcript. Conclusion The clinicians must be vigilant about solely brain metastasis in ARMS without additional metastasis.

Keywords Alveolar rhabdomyosarcoma $\cdot$ Brain metastasis · Children

\section{Introduction}

Rhabdomyosarcoma (RMS), which accounts for 4-10\% of pediatric malignancies, is the most common soft tissue sarcoma of adolescence and childhood $[8,27]$. The annual incidence is approximately 4.5 cases per million in children under the age of 20 [27]. Among all areas in the body, the head and neck region is the most frequent site, accounting for 35-40\% of the cases $[5,6]$.

RMS is a small, round, blue cell tumor usually arising in the skeletal muscle tissue, and it is thought to originate from mesenchymal cells likely committed to the skeletal muscle lineage. Four pathological subtypes have been traditionally defined: alveolar rhabdomyosarcoma, embryonal rhabdomyosarcoma, botyroid rhabdomyosarcoma, and pleiomorphic rhabdomyosarcoma. Embryonal subtype accounts for nearly $70 \%$ with better prognosis, and alveolar subtype accounts for more than $20 \%$ with a relatively poor prognosis. In recent years, the survival rate of RMS has greatly improved with the multimodal therapy proposed by the Intergroup Rhabdomyosarcoma Study 
Group (IRSG); in nonmetastatic RMS, the estimated 5-year overall survival rate was $88 \%$ in the embryonal type and $72 \%$ in the alveolar type $[5,6,8]$. Similar results were reported in Turkey [2]. Alveolar RMS is usually found in adolescents and typically arises in the deep musculature of the extremities while embryonal rhabdomyosarcoma primarily occurs in children younger than 4 years of age [8]. Widespread alveolar RMS metastases to the bone marrow can be present with acute tumor lysis syndrome mimicking hematologic malignancies. These cases present a diagnostic problem to anyone unfamiliar with its clinical features [4, 9-11, 18, 25]. Especially, alveolar RMS with $\mathrm{t}(2 ; 13)$ ( $\mathrm{q} 35 ; \mathrm{q} 14)$ may present with widespread disease, no discernible primary lesion, and, in some cases, may be confined to the marrow $[3,15,16]$.

Although, brain metastasis is frequently encountered in adults with cancer, it is rarely encountered in pediatric solid tumors. Brain metastasis in RMS are generally seen as a leptomeningeal spread after local recurrence or lung metastasis [7].

Here, we describe a case with alveolar rhabdomyosarcoma (ARMS) mimicking hematological malignancies, who presented with brain metastasis 6 months after the completion of chemotherapy.

\section{Case report}

A 14-year-old boy, who had abdominal pain for a week was referred to our hospital because of the enlargement of parainguinal and para-aortic lymph nodes on abdominal ultrasonography and computed tomography. On physical examination, only slight hepatosplenomegaly was found. Although complete blood count was normal, he had an acute renal failure because of tumor lysis syndrome (serum values of BUN, $65 \mathrm{mg} / \mathrm{dl}$; creatinine, $6.3 \mathrm{mg} / \mathrm{dl}$; uric acid, $15 \mathrm{mg} / \mathrm{dl}$; phosphorus, $7.5 \mathrm{mg} / \mathrm{dl}$; and LDH, 2,484 IU/L). Examination of bone marrow aspirate revealed a diffuse infiltration by small, round blast cells with cytoplasmic vacuolation. These blasts were PAS positive. He was suspected with a differential diagnosis of lymphoma with acute tumor lysis syndrome (ATLS) by clinical and biochemical findings and radiological imaging, so steroid treatment was started. PET-CT demonstrated extensive lymphadenopathy and bone marrow involvement. Because response to steroid treatment was not as good as expected, surgical biopsy of the para-aortic lymph node and bone marrow biopsy was performed. Sections of para-aortic lymph node biopsy demonstrated atypical cells with alveolar pattern, narrow cystoplasm, and hyperchromatic uniform nucleus. The immunohistochemistry confirmed a diagnosis of RMS - tumor cells were positive for desmin and vimentin. MR imaging to find the primary tumor show bladder wall thickness and wall irregularity and generalize LAP. Cystoscopy was performed because the bladder can be a primary tumor area. The bladder mucosa was seen as fragile and with no obvious mass. Because of distant metastasis, he was considered at stage IV and at the high-risk group ARMS. He was treated with vincristine, topotecan, and cyclophosphamide for 42 weeks. PET-CT showed no bone marrow involvement or lymphadenopathy at the end of the treatment. Six months after the completion of treatment, the patient was admitted with severe headache, blurred vision, right hemiplegia, and severe bone pain. Cranial magnetic resonance imaging showed multiple hemorrhagic infarctions. Whole body bone scintigraphy and bone marrow aspiration was made because of bone pain. While bone scintigraphy showed diffuse hypermetabolic activity, bone marrow aspiration was normal. Because of the deteriorated general condition with refractory convulsions, the patient was admitted to the pediatric intensive care unit. Brain biopsy was performed. The diagnosis of alveolar rhabdomyosarcoma was confirmed (Fig. 1). On the biopsy material of the brain, the reverse transcriptase polymerase chain reaction (RT-PCR) was performed using one-step RT-PCR kit (QIAGEN One-step RTPCR kit), and the PAX3-FOXO1A fusion transcript was detected (Fig. 2). The patient died 1 day after the biopsy procedure.

\section{Discussion}

Histologically, ARMS tumors typically contain alveoli structures similar in appearance to those seen in the lung though solid variant ARMS do occur. ARMS is associated with a poorer prognosis [16]. Most cases have been associated with advanced RMS presenting with bone marrow (BM) metastases. The clinical picture of a widespread RMS may resemble acute hematologic malignancy. Morandi et al. [18] described a case with RMS that metastasized to the bone marrow presenting as acute leukemia in 1996. There have been case reports of ARMS masquerading as hematologic malignancies, and there have been several cases of ARMS with lymph node involvement. In the absence of an evident primary tumor, the nature of the cells infiltrating the marrow may present a diagnostic problem. RMS with bone marrow involvement but without an obvious primary tumor is easily confused with acute leukemia, particularly of the lymphoblastic type [27].

ARMS with lymph node involvement is a big and challenging diagnostic problem, especially when patients present with clinical and/or laboratory signs of ATLS [4]. Mekni et al. [17] reported a case with ARMS mimicking lymphoma. Also, Hanke et al. [10] reported a 4-year-old boy who presented with an acute swelling at his right groin and increased LDH level, L3 morphology in BM aspirate, and multiple enlarged abdominal lymph nodes. The patient was started with chemotherapy according to the NHL-BFM 95 


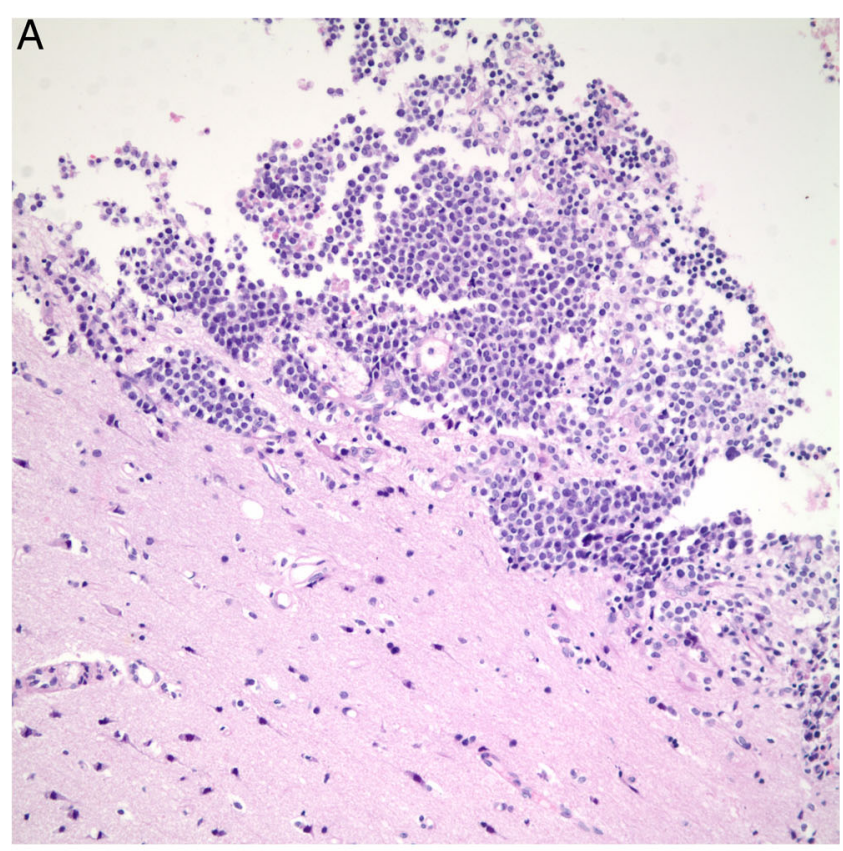

Fig. 2 The gel electrophoresis bands representing PAX3-

FOXO1A gene fusion that were visualized at 145 and $89 \mathrm{bp}$. The band at 89 bp provided a crosscheck for PAX3 involvement

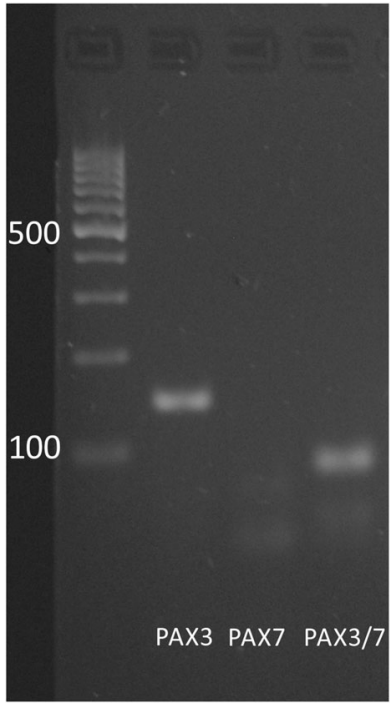

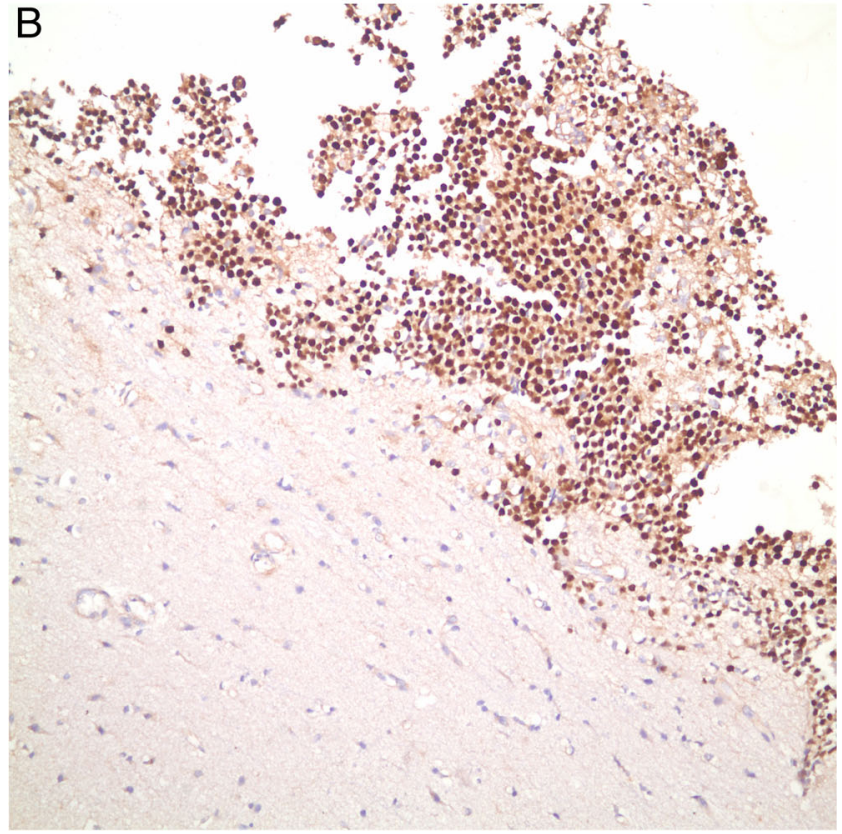

Fig. 1 Hematoxylin eosin staining (a) and immunohistochemical staining of myogenin (b) of ARMS on the brain biopsy of the patient

protocol because of clinical and morphological findings. No clinical response was observed, and lymph node biopsy revealed alveolar RMS.

Despite parenchymal or leptomeningeal metastasis, involvement of the central neurological systems within a child with cancer may be caused by different etiopathogenesis, including paraneoplastic involvement, or neurologic complications resulting from organic and psychotic syndromes related to chemotherapy or radiation treatment and infections originating from immunosuppression related to the disease or its treatment [14, 20-24, 29, 30].
A few similar cases have been reported in the literature in which, after intensive investigation, neither masses nor osteolytic lesions were found at the onset. This lymphadenopathic ARMS shows lymph nodes as a clinical manifestation mimicking lymphoma with an unknown primary neoplasm [18]. Although, a 20-year-old man with ARMS mimicking a lymphoma at presentation was reported by Tsai et al. [28], this case was diagnosed as ARMS because of immunohistochemical staining and cytogenetics.

Our case bears striking similarities to some previously reported cases. It was thought that he had ATLS due to nonHodgkin lymphoma with clinical and biochemical findings. A primary tumor was not found. We have observed that in the $\mathrm{BM}$, biopsy displayed an extensive tumorous infiltrate with the alveolar spaces lined by primitive round cells.

In the subtype of alveolar rhabdomyosarcoma, approximately $80 \%$ of cases harbored two signature chromosomal translocations- $\mathrm{t}(2 ; 13)(\mathrm{q} 35 ; \mathrm{q} 14)$ in $60 \%$ and $\mathrm{t}(1 ; 13)$ (p36;q14) in $20 \%$ of cases. These translocations resulted in the formation and overexpression of chimerical genes PAX3FOXO1 (FOXO1, also known as FKHR) and PAX7-FOXO1, respectively. This aberrant gene expression program is then hypothesized to contribute to tumorigenic behavior by impacting on the control of growth, apoptosis, differentiation, and motility. The aberrant or inappropriate expression of these gene products is postulated to contribute to oncogenic initiation or progression by stimulating cell behaviors such as growth and motility or inhibiting cell behaviors such as apoptosis and terminal differentiation. A characteristic of the ARMS subtype is the occurrence of PAX3-FOXO1 as the most common fusion gene in ARMS. This fusion transcription factor is thought to drive the gene expression that causes the worse prognosis in ARMS $[3,15,16]$. We could not study a chromosomal analysis at the onset. Six months after the completion of chemotherapy, he developed brain metastasis 
without lung metastasis, and, on the brain tissue, $\mathrm{t}(2 ; 13)(\mathrm{q} 35 ; \mathrm{q} 14)$ by molecular analyses were found.

Brain metastasis occurs in $20-30 \%$ of adult patients with cancer, whereas they are uncommon in children. However, as survival increased in pediatric cancer, reports of cases that developed brain metastasis have been increasing. The incidence of brain metastasis in children with solid tumors is $1.5-2.5 \%$ in clinical series and $6-13 \%$ in autopsy series, but varies considerably depending on histology, location, stage, and duration of survival and pattern of systemic metastases [1, 6, 12, 13, 18, 19, 26, 28]. A case of 14-year-old girl is reported in whom an alveolar rhabdomyosarcoma occurred in the soft tissues of the left forearm 4 years prior to death [13].

For brain metastases in children with solid tumors, the presence of lung metastasis appears to be a predisposing factor. Brain metastases most likely arise from hematogenously derived tumor emboli emanating from pulmonary metastasis. Careful examination and imaging studies for neurologic symptoms in children with cancer are important in the diagnosis of brain metastasis [1].

Our patient had brain metastasis from hematogenously derived tumor emboli emanating from unknown other sites without lung metastasis. As a conclusion, the presented case also had a poor prognosis and died after 1 year of diagnosis due to brain metastasis of ARMS mimicking hematological malignancies.

\section{References}

1. Ahola DT, Provenzale JM, Longee DC (1998) Metastatic rhabdomyosarcoma presenting as intracranial hemorrhage: imaging findings. Eur J Radiol 26:241-243

2. Akyüz C, Sari N, Yalçin B, Varan A, Kutluk T, Büyükpamukçu (2012) Long-term survival results of pediatric rhabdomyosarcoma patients: a single-center experience from Turkey. Pediatr Hematol Oncol 29:38-49

3. Barr G (2001) Gene fusions involving PAX and FOX family members in alveolar rhabdomyosarcoma. Oncogene 20:5736-5746

4. Bien E, Maciejka-Kapuscinska L, Niedzwiecki M, Stefanowicz J, Szolkiewicz A, Krawczyk M, Maldyk J, Izycka-Swieszewska E, Tokarska B, Balcerska A (2010) Childhood rhabdomyosarcoma metastatic to bone marrow presenting with disseminated intravascular coagulation and acute tumour lysis syndrome: review of the literature apropos of two cases. Clin Exp Metastasis 27:399-407

5. Chen L, Shah HO, Lin JH (2004) Alveolar rhabdomyosarcoma with concurrent metastases to bone marrow and lymph nodes simulating acute hematologic malignancy. J Pediatr Hematol Oncol 26:696-697

6. Chen SC, Bee YS, Lin MC, Sheu SL (2011) Extensive alveolar-type paranasal sinus and orbit rhabdomyosarcoma with intracranial invasion treated successfully. J Chin Med Assoc 74:140-143

7. Chou YS, Liu CY, Chen WM, Chen TH, Chen PC, Wu HT, Shiau CY, Wu YC, Liu CL, Chao TC, Tzeng CH, Wong TT, Yen CC (2011)
Brain, the last fortress of sarcoma: similar dismal outcome but discrepancy of timing of brain metastasis in bone and soft tissue sarcoma. J Surg Oncol 104:765-70

8. Crist WM, Anderson JR, Meza JL, Fryer C, Raney RB, Ruymann FB, Breneman J, Qualman SJ, Wiener E, Wharam M, Lobe T, Webber B, Maurer HM, Donaldson SS (2001) Intergroup rhabdomyosarcoma study-IV: results for patients with nonmetastatic disease. J Clin Oncol 19:3091-3102

9. Ganesan P, Thulkar S, Rajan A, Bakhshi S (2008) Solid variant of alveolar rhabdomyosarcoma mimicking non-Hodgkin lymphoma: case report and review of literature. J Pediatr Hematol Oncol 30: 772-774

10. Hanke CA, Roessler J, Stegmaier S, Koscielniak E, Niemeyer CM, Kontny U (2007) Alveolar rhabdomyosarcoma mimicking lymphoma with bone marrow involvement. Eur J Pediatr 166: 505-506

11. Jani P, Charles CY (2009) Massive bone marrow involvement by clear cell variant of rhabdomyosarcoma. Indian J Pediatr 76:224-228

12. Kebudi R, Ayan I, Görgün O, Ağaoğlu FY, Vural S, Darendeliler E (2005) Brain metastasis in pediatric extracranial solid tumors: survey and literature review. J Neurooncol 71:43-48

13. Kleinert R, Beham A, Rosanelli G (1998) Alveolar rhabdomyosarcoma in a young female patient metastasizing to the brain. Acta Neuropathol 67:341-344

14. Kose D, Paksoy Y, Koksal Y, Unal E (2014) Neurological complication of non Hodgkin lymphoma in childhood: experience from a single center in Turkey. Childs Nerv Syst 30: 639-645

15. Liu J, Guzman MA, Pezanowski D (2011) FOXO1-FGFR1 fusion and amplification in a solid variant of alveolar rhabdomyosarcoma. Mod Pathol 24:1327-1335

16. Marshall AD, Grosveld GC (2012) Alveolar rhabdomyosarcomathe molecular drivers of PAX3/7-FOXO1-induced tumorigenesis. J Skelet Muscle 2:25

17. Mekni A, Bouraoui S, Boussen H, el May A, Kchir N (2004) Lymphadenopathic form of alveolar rhabdomyosarcoma: a case report. Tunis Med 82:241-244

18. Morandi S, Mana A, Sabattini E, Porcellini A (1996) Rhabdomyosarcoma presenting as acute leukemia. J Pediatr Hematol Oncol 18:305-307

19. Ogose A, Morita T, Hotta T, Kobayashi H, Otsuka H, Hirata Y, Yoshida S (1999) Brain metastases in musculoskeletal sarcomas. Jpn J Clin Oncol 29:245-247

20. Ozdemir MA, Torun YA, Karakukcu M, Kontas O, Patiroglu T, Yikilmaz A (2011) Lymphomatoid granulomatosis in 10-month-old infant with lymphopenia: case report and review of the literature. UHOD 21:124-128

21. Ozbek O, Koc O, Paksoy Y, Aydin K, Nayman A (2011) EpsteinBarr virus encephalitis: findings of MRI, MRS, diffusion and perfusion. Turk J Pediatr 53:680-683

22. Ozyurek E, Ozbek N (2011) Accidental intrathecal methotrexate overdose. Turk J Hematol 28:245-246

23. Patiroglu T, Unal E, Karakukcu M et al (2012) Multiple fungal brain abscesses in a child with acute lymphoblastic leukemia. Mycopathologia 174:505-509

24. Patiroglu T, Unal E, Yikilmaz A, Koker MY, Ozturk MK (2010) Atypical presentation of chronic granulomatous disease in an adolescent boy with frontal lobe located Aspergillus abscess mimicking intracranial tumor. Childs Nerv Syst 26:49-54

25. Prashant J, Ye C (2009) Massive bone marrow involvement by clear cell variant of rhabdomyosarcoma. Indian J Pediatr 76:224-225

26. Rodriguez-Galindo C, Hill DA, Onyekwere O, Pin N, Rao BN, Hoffer FA, Kun LE, Pappo AS, Santana VM (2001) Neonatal alveolar rhabdomyosarcoma with skin and brain metastases. Cancer 92: $1613-1620$ 
27. Sandberg AA, Stone JF, Czarnecki L, Cohen JD (2001) Hematologic masquerade of rhabdomyosarcoma. Am J Hematol 68:51-57

28. Tsai SC, Reale LD, Flomenberg N, Schwarting R, Enck RE (2006) Alveolar rhabdomyosarcoma mimicking a lymphoma at presentation. J Clin Oncol 24:4031-4032
29. Unal E, Koksal Y, Baysal T, Energin M, Aydin K, Caliskan U (2007) Kluver-Bucy syndrome in a boy with non-Hodgkin lymphoma. Pediatr Hematol Oncol 24:149-152

30. Unal E, Yazar A, Koksal Y, Caliskan U, Paksoy Y, Kalkan E (2008) Cerebral venous sinus thrombosis in an adolescent with Ewing sarcoma. Childs Nerv Syst 24:983-986 